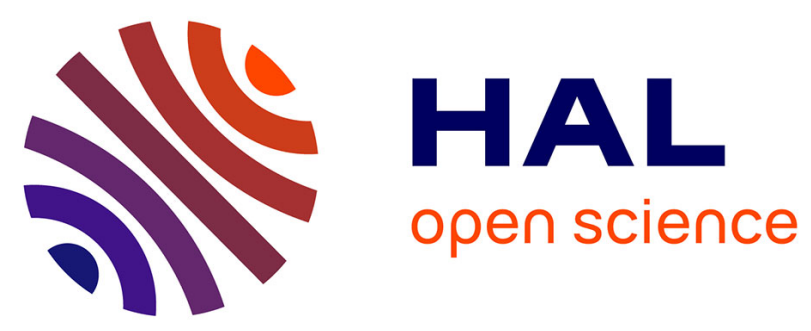

\title{
ÉTUDE DE TRANSITIONS \\ ÉLECTROMAGNÉTIQUES PAR DES MESURES DE DISTRIBUTION-POLARISATION ET DE CORRÉLATION ANGULAIRE $\gamma-\gamma$
}

S. Gorodetzky, F. Beck, R. Bertini, E. Bożek, Jacques Chevallier, A. Knipper

\section{To cite this version:}

S. Gorodetzky, F. Beck, R. Bertini, E. Bożek, Jacques Chevallier, et al.. ÉTUDE DE TRANSITIONS ÉLECTROMAGNÉTIQUES PAR DES MESURES DE DISTRIBUTION-POLARISATION ET DE CORRÉLATION ANGULAIRE $\gamma$ - $\gamma$. Journal de Physique Colloques, 1966, 27 (C1), pp.C1-112-C1114. 10.1051/jphyscol:1966156 . jpa-00213040

\author{
HAL Id: jpa-00213040 \\ https://hal.science/jpa-00213040
}

Submitted on 1 Jan 1966

HAL is a multi-disciplinary open access archive for the deposit and dissemination of scientific research documents, whether they are published or not. The documents may come from teaching and research institutions in France or abroad, or from public or private research centers.
L'archive ouverte pluridisciplinaire HAL, est destinée au dépôt et à la diffusion de documents scientifiques de niveau recherche, publiés ou non, émanant des établissements d'enseignement et de recherche français ou étrangers, des laboratoires publics ou privés. 


\title{
ÉTUDE DE TRANSITIONS ÉLECTROMAGNÉTIQUES PAR DES MESURES DE DISTRIBUTION-POLARISATION ET DE CORRÉLATION ANGULAIRE $\gamma-\gamma$
}

\author{
S. Gorodetzky, F. BeCK, R. Bertini, E. BożeK $\left({ }^{1}\right)$, \\ J. Chevallier et A. KNIPPer \\ Centre de Recherches Nucléaires \\ Département de Physique nucléaire \\ Strasbourg-Cronenbourg
}

\begin{abstract}
Résumé. - Les transitions électromagnétiques de niveaux alignés peuvent être étudiées sans connaissance détaillée du procédé d'alignement (réaction nucléaire). Deux méthodes sont utilisées: a) comparaison de la distribution angulaire et de la polarisation plane des rayons gamma ; $b$ ) corrélation angulaire gamma-gamma suivant la méthode indiquée par Litherland et Ferguson. L'appareillage est décrit sommairement. Les résultats concernent des transitions dans $12 \mathrm{~B}(955 \mathrm{keV})$, ${ }^{13} \mathrm{C}(170 \mathrm{keV})$ et ${ }^{53} \mathrm{Mn}(380,910$ et $1290 \mathrm{keV})$.
\end{abstract}

Abstract. - Electromagnetic transitions from aligned levels can be studied without a detailed knowledge of the alignment process (nuclear reaction). Two methods are used : $a$ ) comparison of the angular distribution and plane polarization of the gamma rays; b) gamma-gamma angular correlations as suggested by Litherland and Ferguson. The apparatus is briefly described, together with results for transitions in ${ }^{12 \mathrm{~B}}(955 \mathrm{keV}),{ }^{13} \mathrm{C}(170 \mathrm{keV})$ and ${ }^{53 \mathrm{Mn}}(380,910$ and $1290 \mathrm{keV})$.

I. Formalisme. - Les niveaux excités de faible énergie ont été étudiés par l'observation des rayons $\gamma$ émis, en vue de déterminer les moments angulaires et les coefficients de mélange $\delta$. Deux méthodes de corrélation angulaire ont été utilisées qui ne nécessitent pas une description détaillée des réactions nucléaires $[1,2]$ :

1) Corrélations doubles (Méthode de distribution-polarisation).

Le processus peut être résumé par la séquence $i(R) a(\gamma) b$. La première étape est une absorption (ou une émission) de rayonnements dans une direction déterminée, fixant un axe de quantification, et éventuellement des radiations inobservées. La deuxième est une transition électromagnétique.

Expérimentalement, nous mesurons la fonction de distribution angulaire

$$
\begin{aligned}
W(\theta) & =\sum_{k} A_{k} \cdot P_{k}(\cos \theta) \\
A_{k} & =A_{k}(R) \cdot A_{k}(\gamma)
\end{aligned}
$$

et la polarisation plane $p$ des rayons $\gamma$ émis. Les facteurs $A_{k}(R)$, proportionnels aux tenseurs statistiques du niveau $a$, peuvent être éliminés entre les expressions de $W(\theta)$ et $p$. Ceci conduit à la relation semi-empirique $[4,5]$ :

$$
p . W(\theta)=Y\left(a, b ; \sigma ; \delta ; A_{k}\right) .
$$

La forme de la fonction $Y$ est connue : elle contient les coefficients $F_{k}$ tabulés [3], et dépend de la parité $\sigma$ de la radiation.

La figure 1 représente une résolution graphique de cette relation.

2) CoRrélations tRIPLES (Corrélations $\gamma-\gamma$ avec niveau initial orienté).

La séquence est $i(R) a(1) b(2) c$, et comprend l'orientation nucléaire $R$ suivie d'une émission en cascade de deux rayons gamma. La fonction de corrélation triple peut être développée en polynômes angulaires connus :

$$
W\left(\theta_{1}, \theta_{2}, \varphi\right)=\sum_{K M N} a_{K M}^{N} \cdot P_{K M}^{N}\left(\theta_{1}, \theta_{2}, \varphi\right) .
$$

Les coefficients de (3) dépendent de facteurs tabulés $[6,7]$, faisant intervenir les moments angulaires, des populations $P(m)$ du niveau $a$ (ou des tenseurs statistiques) qui sont maintenant des paramètres à déterminer expérimentalement, et des coefficients de mélange $\delta_{1}$ et $\delta_{2}$.

(1) Adresse permanente : Institut Fizyki Jadrowej, Kraków. 


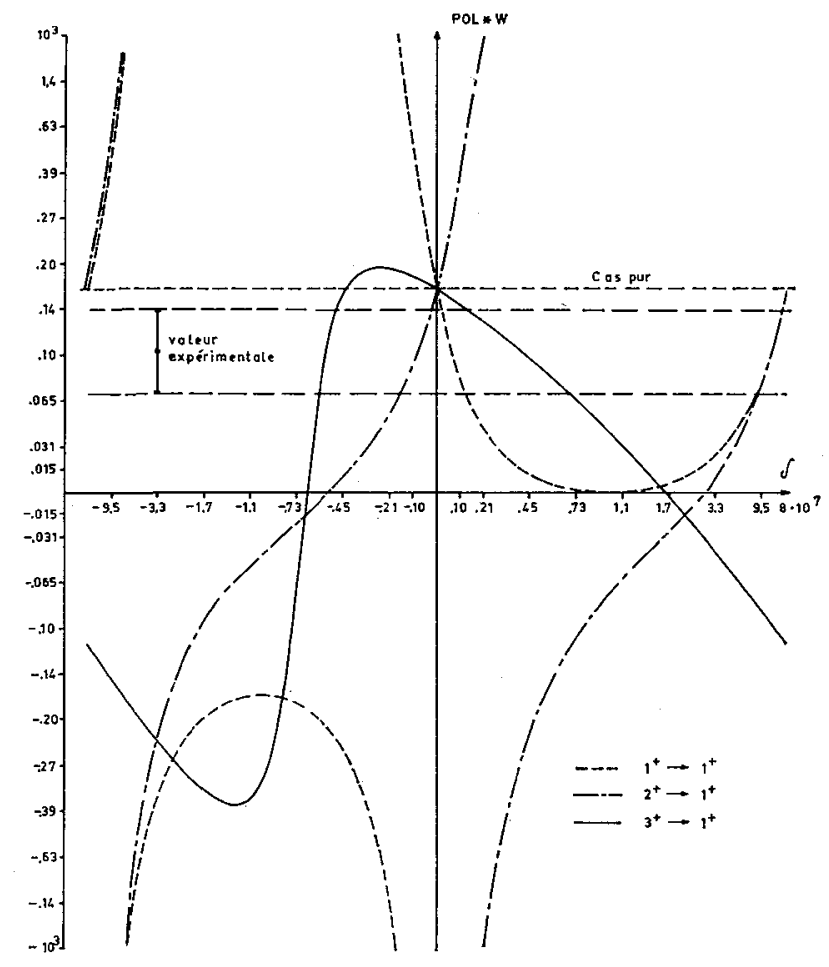

FIG. 1. - Variations de $p(\theta) . W(\theta)$ en fonction du coefficient de mélange. Réaction ${ }^{11} \mathrm{~B}(\mathrm{~d}, \mathrm{p}){ }^{12 \mathrm{~B}}$ pour $E_{\mathrm{d}}=700 \mathrm{keV}$.

Litherland et Ferguson [8] ont proposé d'étudier les fonctions du type (3) en ne faisant varier qu'un seul angle, appelons-le $\alpha$, et de donner aux deux autres les valeurs $0^{\circ}$ ou $90^{\circ}$. On obtient sept fonctions indépendantes (“ géométries 》).

$$
W_{g}(\alpha), g=1, \ldots, 7 \text {. }
$$

Notre méthode d'analyse consiste à comparer les coefficients expérimentaux $a_{i}$ des géométries (4) mesurées, développées en polynômes de Legendre directs, à leurs équivalents théoriques $b_{i}$, en formant l'expression

$$
\chi^{2}=\sum_{i j}\left(a_{i}-b_{i}\right)\left(a_{j}-b_{j}\right) X_{i j}^{-1}
$$

où $X_{i j}$ est la matrice des erreurs corrélées. Pour des moments angulaires de $a, b, c$ fixés, nous minimisons en chaque point du plan $\left(\delta_{1}, \delta_{2}\right)$ l'expression (5) par rapport aux populations $P(m)$. Les valeurs de (5), calculées pour les populations ainsi déterminées, sont tabulées sous le nom

$$
Z\left(\delta_{1}, \delta_{2}\right) \text {. }
$$

Les minimums de la surface (6) constituent les solutions cherchées (voir Fig. 2), et leur probabilité est obtenue à l'aide d'une table des $\chi^{2}$.

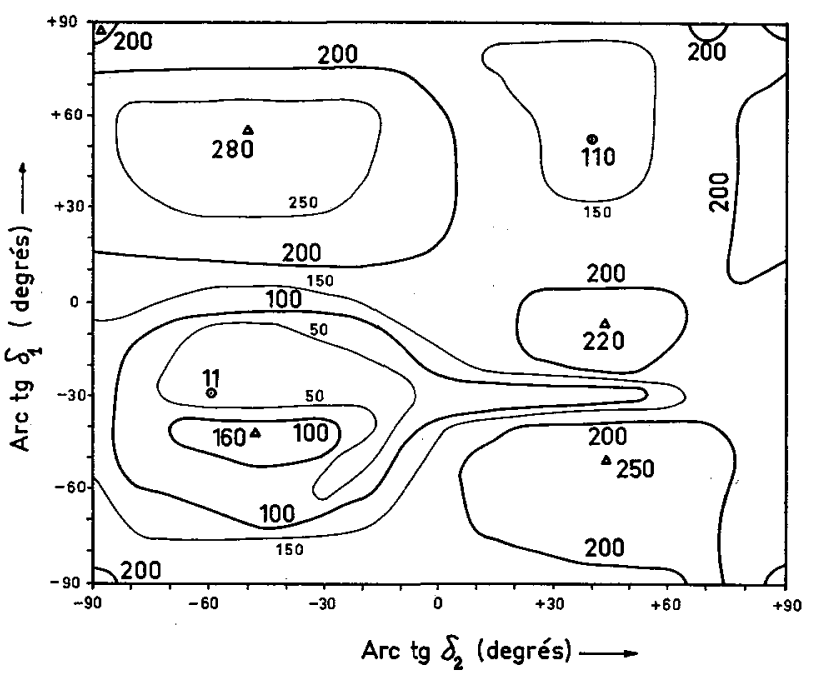

FIG. 2. - Recherche des solutions par le tracé des lignes de niveaux de la surface $Z\left(\delta_{1}, \delta_{2}\right)$. Cas des géométries II et III de l'expérience du $53 \mathrm{Mn}$, pour la cascade $3 / 2-5 / 2-7 / 2$. Les sommets sont marqués d'un triangle et les points les plus bas d'un cercle. Les chiffres correspondent à $100 . \log _{10}(Z)$.

II. Appareillage. - Toutes les mesures sont faites en spectre, en enregistrant sur un sélecteur multicanaux les spectres de deux ou plusieurs compteurs. Un ordinateur calcule l'aire des pics et effectue tous les traitements numériques ultérieurs; un calcul d'erreur accompagne chaque opération.

1) Distributions et Corrélations angulaires. Des compteurs $\mathrm{NaI} 1^{\prime \prime} 3 / 4 \times 2^{\prime \prime}$ de bonne résolution (Scintibloc Quartz et Silice) sont utilisés. Le centrage est corrigé en mesurant des distributions angulaires isotropes.

Pour les distributions angulaires, un compteur fixe contrôle le compteur mobile, monté sur le plateau mobile d'un goniomètre.

Pour mesurer simultanément 4 géométries de corrélation angulaire, nous disposons de deux compteurs fixes à $90^{\circ}$ du faisceau, l'un FH dans le plan du compteur mobile, l'autre FV perpendiculaire au plan. Le compteur mobile est analysé par la méthode de Hoogenboom, en coïncidence avec chacun des compteurs fixes [9].

2) Polarimètre. C'est un polarimètre Compton, utilisant un petit diffuseur $\mathrm{D}$ de INa et deux analyseurs fixes : $H$ dans le plan de réaction, $V$ perpendiculaire au plan $[4,5]$. L'asymétrie instrumentale est mesurée à $0^{\circ}$ (polarisation nulle) ou à l'aide de sources. La variation en fonction de l'énergie de l'efficacité d'analyse $\pi_{1}$ a été calculée pour diverses géométries, et peut être calibrée à l'aide de rayons $\gamma$ fortement polarisés obtenus par 
Tableau I

\begin{tabular}{|c|c|c|c|c|c|c|}
\hline Niveau & $E_{\gamma}(\mathrm{keV})$ & Réaction & $10^{3} A_{2}$ & ${ }^{103} p$ & $10^{3} \delta$ & \\
\hline $13 \mathrm{C}, 5 / 2^{+}, 3.85 \mathrm{MeV}$ & 170 & ${ }^{12} \mathrm{C}(\mathrm{d}, \mathrm{p}) 2,490 \mathrm{MeV}$ & $-356 \pm 10$ & $-584 \pm 65$ & $35 \pm 17$ & cible mince \\
\hline & & & $-269 \pm 10$ & $-453 \pm 46$ & $33 \pm 14$ & cible épaisse \\
\hline $12 \mathrm{~B}, 2^{+}, 955 \mathrm{keV}$ & 955 & $11 \mathrm{~B}(\mathrm{~d}, \mathrm{p}) 700 \mathrm{keV}$ & $-113 \pm 7$ & $+101 \pm 29$ & $-85 \pm 63$ & (autres solutions) \\
\hline $53 \mathrm{Mn}, 5 / 2^{-}, 380 \mathrm{keV}$ & 380 & $\left.\begin{array}{l}{ }_{52 \mathrm{Cr}}(\mathrm{p}, \gamma) \\
53 \mathrm{Cr}(\mathrm{p}, \mathrm{n})\end{array}\right\} 3,15 \mathrm{MeV}$ & $-125 \pm 6$ & $-6 \pm 8$ & \multirow{3}{*}{\multicolumn{2}{|c|}{ nombreuses solutions }} \\
\hline $53 \mathrm{Mn}, 3 / 2^{-}, 1290 \mathrm{keV}$ & 910 & ${ }^{53} \mathrm{Cr}(\mathrm{p}, \mathrm{n}) \quad 3,25 \mathrm{MeV}$ & $-57 \pm 29$ & $-132 \pm 58$ & & \\
\hline $53 \mathrm{Mn}, 3 / 2^{-}, 1290 \mathrm{keV}$ & 1290 & ${ }^{53} \mathrm{Cr}(\mathrm{p}, \mathrm{n}) \quad 3,25 \mathrm{MeV}$ & $-52 \pm 29$ & $+131 \pm 81$ & & \\
\hline
\end{tabular}

Tableau I : Résultats de quelques expériences de distribution - polarisation dans les noyaux légers.

Les valeurs de $A_{4}$ sont statistiquement nulles.

des réactions $\left(p p^{\prime}\right)$. La résolution en énergie a été considérablement améliorée en mesurant les spectres de somme, $\mathrm{D}+\mathrm{H}$ et $\mathrm{D}+\mathrm{V}$, plutôt que les spectres de l'un ou l'autre compteur.

III. Résultats expérimentaux. - Le tableau I montre les résultats de plusieurs mesures de distributionpolarisation. L'expérience concernant le ${ }^{13} \mathrm{C}$ a été motivée par la forte interdiction de la transition $E 1$. L'expérience $\mathrm{du}{ }^{12} \mathrm{~B}$ nous a servi à mettre au point cette méthode. Les moments angulaires $J_{a}=0^{+}, 1^{+}$, $2^{+}, 3^{+}$sont a priori possibles. La valeur $J_{a}=0^{+}$ est exclue (isotropie) et la valeur $3^{+}$très peu probable (fort mélange quadrupôle-octupôle), contredisant une proposition récente [10]. La valeur $J_{a}=2^{+}$serait en accord avec le modèle en couches, les largeurs réduites de la réaction ${ }^{11} B(d, p){ }^{12} B$ et la position du premier niveau $2^{+}, T=1$ dans le ${ }^{12} \mathrm{C}$; la mesure de vie moyenne [11] n'autorise que la plus petite solution pour le coefficient de mélange.

Dans le cas du ${ }^{53} \mathrm{Mn}[4,5]$, on observe les rayons $\gamma_{1}(910 \mathrm{keV})$ et $\gamma_{2}(380 \mathrm{keV})$ en cascade, et le rayon direct $\gamma_{3}$, dans la réaction ${ }^{53} \mathrm{Cr}(\mathrm{p}, \mathrm{n}){ }^{53} \mathrm{Mn}$. La valeur $J_{c}=7 / 2^{-}$est connue. Nous avons fait des expériences de distribution-polarisation pour $\gamma_{1}, \gamma_{2}$ et $\gamma_{3}$ et mesuré plusieurs géométries dans la cascade $\gamma_{1}-\gamma_{2}$. La combinaison des divers résultats et l'utilisation de la valeur expérimentale du rapport des intensités $\gamma_{1} / \gamma_{3}$ conduit à la solution unique $[4,9]$ :

$$
J_{a}=3 / 2^{-} ; J_{b}=5 / 2^{-} \text {. }
$$

Ces résultats s'expliquent bien si les trois niveaux appartiennent à la configuration $(1 f 7 / 2)_{J}^{3}$.
Une expérience analogue est en cours, en vue de l'étude des niveaux $\mathrm{du}{ }^{48} \mathrm{Sc}$ dans la réaction ${ }^{48} \mathrm{Ca}(\mathrm{p}, \mathrm{n}){ }^{48} \mathrm{Sc}$.

Une mesure de corrélation triple dans le ${ }^{13} \mathrm{C}$ a été abordée, dans le but de mesurer le coefficient de mélange de la transition de $3,68 \mathrm{MeV}$.

\section{Bibliographie}

[1] Voir par exemple les articles de H. Frauenfelder et R. M. Steffen et S. R. de Groot, H. A. ToLHoek et W. J. Huiskamp, dans le Chapitre XIX de "Alpha-, Beta- and Gamma-Ray Spectroscopy ", édité par K. Siegrahn, Amsterdam, 1965.

[2] Ferguson (A. J.), Angular Correlation Methods in Gamma-Ray Spectroscopy, Amsterdam, 1965.

[3] Ferentz (M.) et Rosenzweig (N.), A. N. L. Report $\mathrm{N}^{\circ} 5324,1955$.

[4] BeCK (F.), Thèse de Doctorat d'Etat, Strasbourg, 1965.

[5] Gorodetzky (S.), Beck (F.), Bertini (R.), Chevalurer (J.) et KnIPPer (A.), Nucl. Phys. (à paraître).

[6] Sмith (P. B.), Nuclear Reactions II, édité par P. M. ENDT et P. B. SMITH, Amsterdam, 1962.

[7] Kaye (G.), Read (E. J. C.) et Willmott (J. C.), Tables of Coefficients for the Analysis of Triple Angular Correlations from Aligned Nuclei, University of Liverpool, 1963.

[8] Litherland (A. E.) et Ferguson (A. J.), Can. $J$. Phys., 1961, 39, 788.

[9] Gorodetzky (S.), Beck (F.), Bertini (R.), Bozek (E.) et KnipPer (A.), Nucl. Phys. (à paraître).

[10] Middleton (R.) et Pullen (D. J.), Nucl. Phys., 1964, 51, 50.

[11] Warburton (E. K.) et Chase (L. F.), Phys. Rev,, 1963, 13, 22273. 